\title{
PERFORMANCE OF RING FOOTING RESTING ON REINFORCED MULTILAYERED SOIL USING PLAXIS 2D
}

\author{
A.I. Dhatrak ${ }^{1}$, P.C. Mishra ${ }^{2}$ \\ ${ }^{1}$ Associate professor, Civil Engineering Department, Government College of Engineering Amravati , Maharashtra, \\ India \\ ${ }^{2}$ M.Tech Student, Civil Engineering Department, Government College of Engineering Amravati, Maharashtra, India
}

\begin{abstract}
Ring footings are efficiently and economically applied as foundation for axis symmetric structures. This paper serves a numerical study to find out the performance of model ring footing resting on reinforced multilayered soil. The software PLAXIS 2D is used for the analysis which works on one of the best method of numerical analysis i.e. Finite element Method. Geogrid is used as soil reinforcement. The analysis was based on Mohr-Coulomb's criterion for soil. The inner to outer diameter ratio of ring footing chosen are 0.2, 0.4,0.6 and 0.8. The parameters considered for analysis are Geogrid number of layers, soil layers and different inner to outer diameter ratio of ring footing are investigated. The ultimate bearing capacity and settlement reduction was calculated for different number of reinforcement layers and radius ratio. From it is found that there is a considerable improvement in load carrying capacity and increase in settlement reduction ratio when reinforcement is provided in layers. The load carrying capacity of ring footing is found to be maximum at a radius ratio 0.4 . For this radius ratio, when the reinforcement is provided in single layer, the bearing capacity improvement is found to be $110 \%$. For two layer and three layer reinforcement $250 \%$ and $300 \%$ bearing capacity improvement is noticed for radius ratio 0.4 . For fourth to seventh the improvement in the bearing capacity is around 10\%. The settlement reduction is found to be around 40 to $50 \%$ for three layer reinforcement and for further reinforcement layer settlement reduction is around $5 \%$.
\end{abstract}

Keywords: Ring Footing, Multilayered Soil, Geogrid, Bearing Capacity, Settlement Reduction Ratio

\section{INTRODUCTION}

Ring footings are economical and effective for axisymmetric structures having circular cross setion. With the increase in use of these foundations need for investigating its behavior has become important. The behavior includes the load displacement response and ultimate bearing capacity. Normally soil exists in the different layer in nature, it does not exit in homogeneous nature we generally assume. The behavior of footing on multilayered soil is studied by various researchers. The innovative technique of reinforcing the soil by the use of geosynthetics is one of the most economical solutions comparing to the use of deep foundations. Previous studies indicated that reinforcing soil with geogrid led to a notable increase in ultimate load carrying capacity of low depth foundations with different shapes.

In this paper the computational analysis of ring footing resting on three layered soil with geogrid reinforcement is studied.

\subsection{Background}

As per studies of rearchers the behavior of ring footings experimental, numerically \& by computational modeling with the use of softwares like PLAXIS 2D. Sawwaf and Nazir [1] performed practical study to find out the performance of not concentric loaded model ring footing situating on a dense layer of sand that rested on layer of loose sand and come to the conclusion that the maximum bearing capacity was at $r_{i} / r_{o} \quad 0.49$. Boushehrian and Hataf [3] performed experiments on ring footing resting on reinforced clay and evaluated the load carrying capacity of footing. Al- Sanad et al. [2] and Ismael [4] conducted the tests on both circular and ring plates in denser calcium content and compacted sands. Ohri et al. [5] conducted experimental tests on ring footings of various radius ratio and conluded that 0.48 diameter ratio is optimum. Hataf and Razavi[6] investigated that radius ratio $0.2-0.4$ is better from bearing capacity poit of view.

Several researchers studied numerically the ring footing resting on different types of sand and conluded the maximum load carrying capacity and displacement . (Choobbasti et al [7]; Ziaie Moayed et al[8]; Zhao and Egorov [9]; Wang[10]; Kumar and Ghosh [11]; Bowles [12];Zhao and Wang [13];). Egorov [9] gave numerical relationships to evaluate the load carrying pressure and settlement under the ring footing. Choobbasti et al [7] analysed the ring footing using PLAXIS software for calculating the bearing capacity and settlement of ring footing. They concluded that there is linear relationship between bearing capacity and depth of footing and performance of ring footing does not largly differs from strip footing as radius ratio increases. Kumar and Ghosh [11] studied the bearing-capacity factor $\mathrm{N} \gamma$ for both smooth and rough ring footings using the method of characteristics. 


\section{COMPUTATIONAL MODELING}

The modeling relating to computation is processed using the PLAXIS 2D software. For the accurate calculations of the stress and strains the 15 noded element and finer mesh is used for modelling. The evaluation of load carrying capacity was done on a 60 meter thickness of soil. The outer radius of ring footing is 15 meters and the inner radiuses are varied as 3, 6, 9 and 12 meters. Figure (1) represents model for the calculations for load carrying capacity and shows the fixity assumed at base and boundaries at the sides.

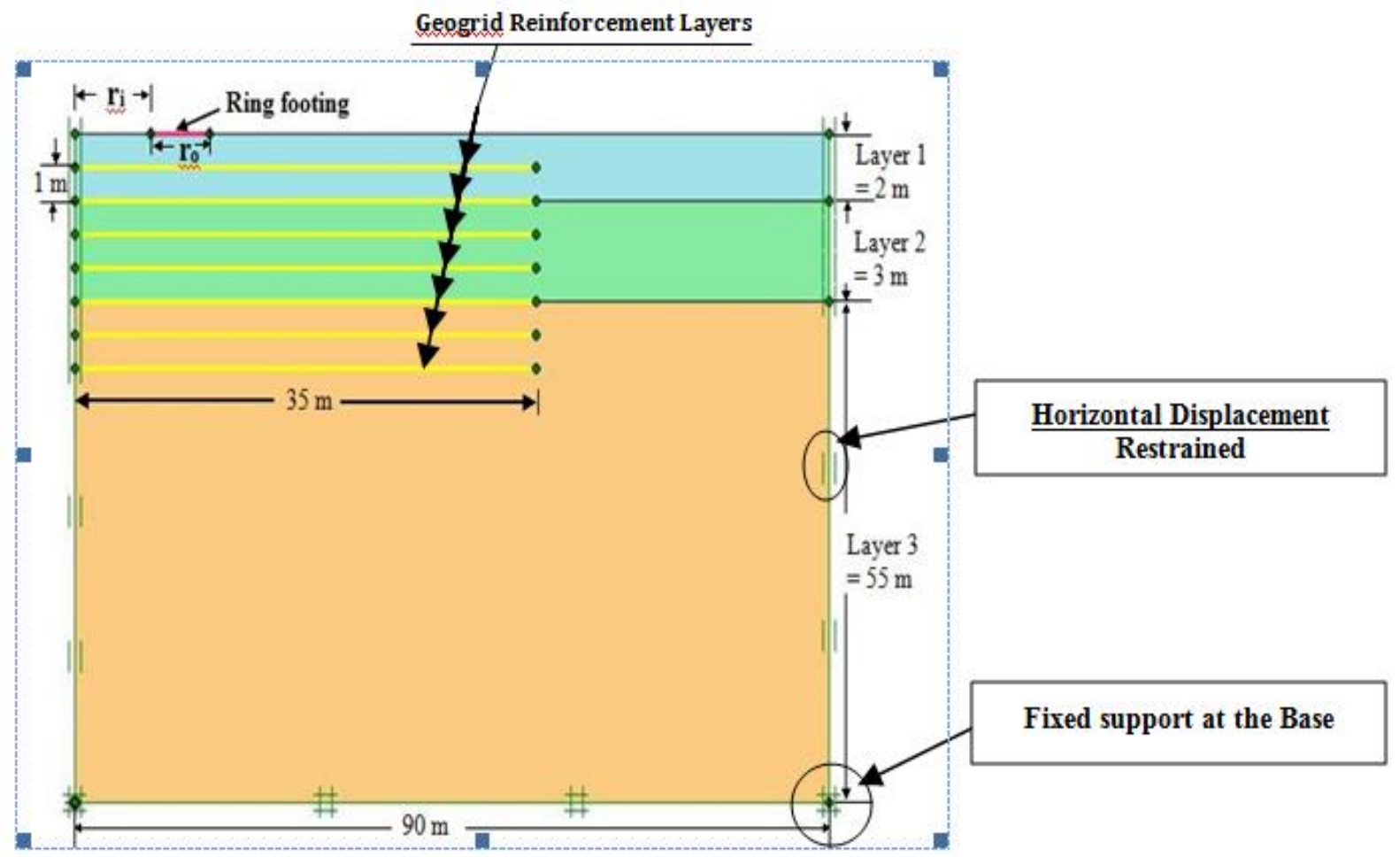

Fig 1. Numerical Model for the Bearing Capacity Calculations.

Table (1) represents the parameters used for the evaluation of load carrying capacity of the three different soil layer i.e. Strong, medium, soft layered soils.

Table 1. Soil parameters/properties

\begin{tabular}{|c|c|c|c|}
\hline Parameters & Soft & Medium & Strong \\
\hline Soil & $\mathrm{c}-\phi$ & $\mathrm{c}-\phi$ & $\mathrm{c}-\phi$ \\
\hline Material model & Mohr Coulomb & Mohr Coulomb & Mohr Coulomb \\
\hline Type of material behavior & Drained & Drained & Drained \\
\hline Unit weight above phreatic level $(\gamma \mathrm{unsat}, \mathrm{kN} / \mathrm{m} 3)$ & 17 & 16 & 20 \\
\hline Unit weight below phreatic level $(\gamma \mathrm{sat}, \mathrm{kN} / \mathrm{m} 3)$ & 20 & 18 & 40000 \\
\hline Young's modulus $(\mathrm{E}, \mathrm{kN} / \mathrm{m} 2)$ & 13000 & 20000 & 1 \\
\hline Cohesion $(\mathrm{cref}, \mathrm{kN} / \mathrm{m} 2)$ & 17 & 5 & 36 \\
\hline Friction angle $(\phi, \mathrm{o})$ & 15 & 28 & 0.4 \\
\hline Poisson's ratio $(v)$ & 0.4 & 0.45 & 3 \\
\hline Dilatancy Angle $(\varphi, \mathrm{o})$ & 0 & 0 & \\
\hline
\end{tabular}




\section{UNREINFORCED AND REINFORCED SOIL}

Following figure shows the concept of failure for the unreinforced soil below ring footing.

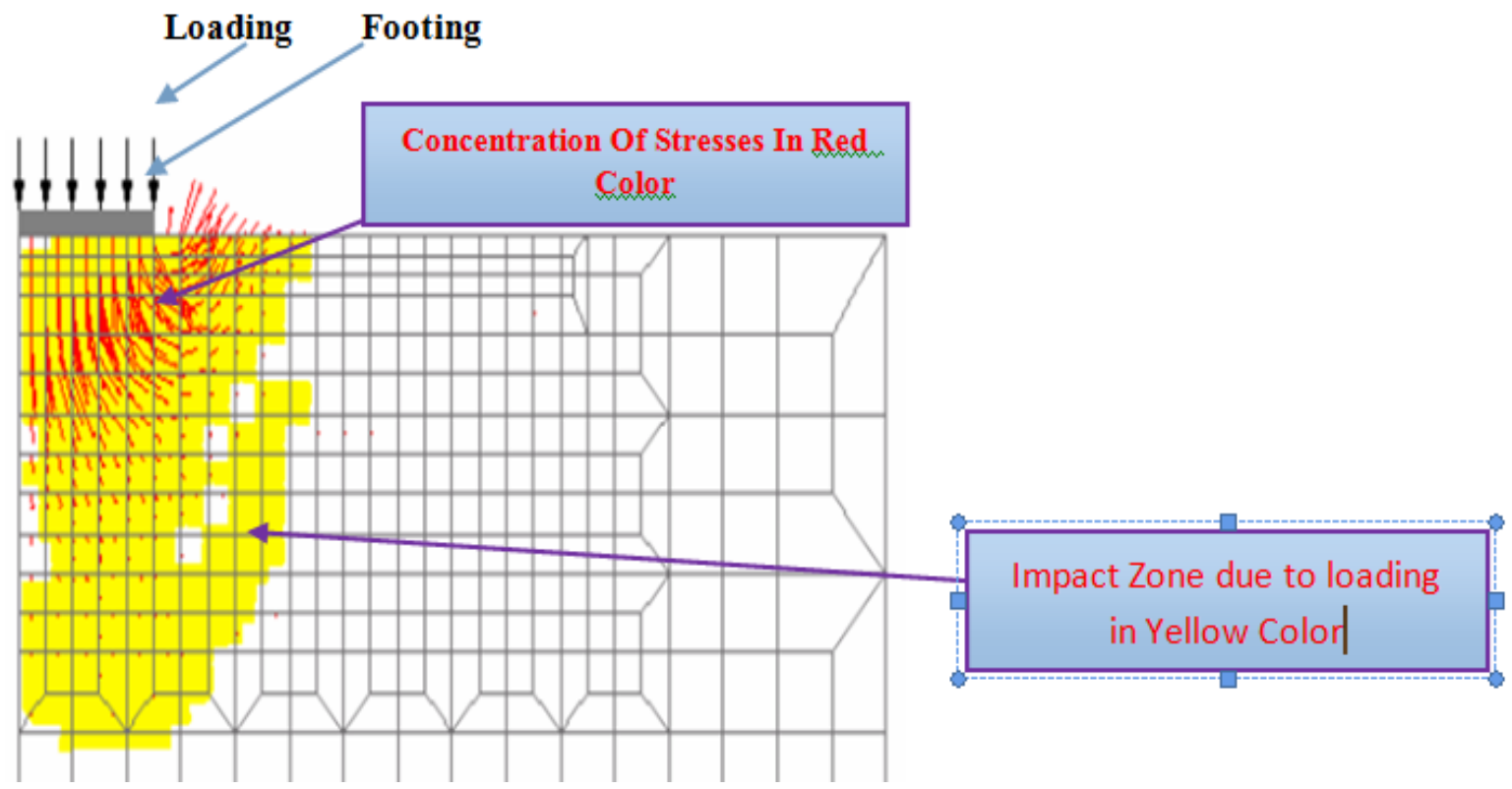

Figure 2: Failure Concept - Unreinforced Soil.

The load carrying capacity of ring footing resting on a layer of geogrid below for one radius ratio under axisymmetric condition. The bearing capacity improvement is calculated by the bearing capacity ratio (BCR) is formulated as:

$$
\mathrm{BCR}=\frac{\kappa}{\kappa_{\mathrm{ult}}^{0}}
$$

Where,

$\kappa$ factor is load carrying capacity of ring footing for particular number layer of reinforcement and radius ratio.

And $\kappa^{0}{ }_{\text {ult }}$ is load carrying capacity of ring footing for unreinforced soil bed.

For consistency the BCR must be calculated at a particular settlement level.

The settlement reduction ratio (SRR) is formulated as:

$\operatorname{SRR}(\%)=\left[\frac{\delta^{0}-\delta^{\mathrm{r}}}{\delta^{0}}\right] 100$

where, $\delta^{0}$ is the settlement at the ultimate load of unreinforced foundation soil and $\delta^{\mathrm{r}}$ is the settlement of reinforced foundation soil at the ultimate load of unreinforced soil foundation. Figure 3 illustrates these indexes.

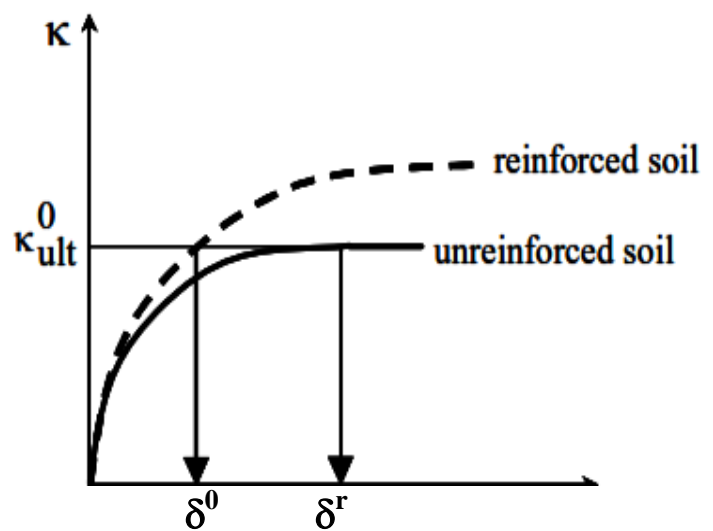

Figure 3: Reference Parameters $\delta^{0} \& \delta^{\rho}$ Definition.

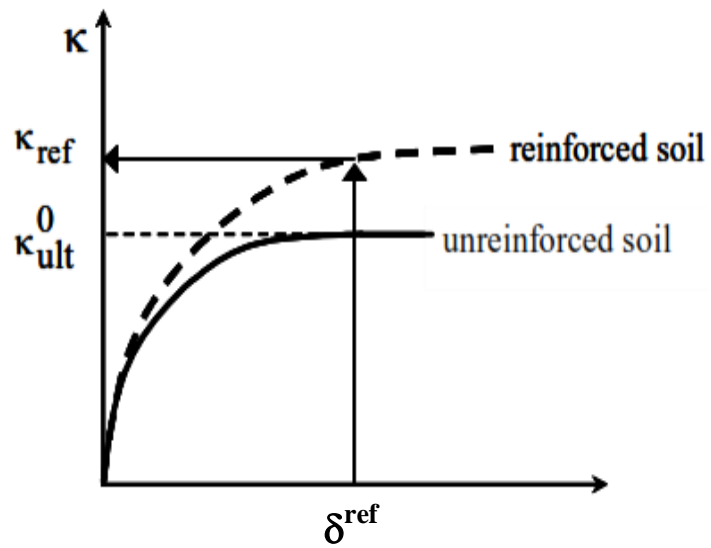

Figure 4: Reference Parameters $\mathrm{k}_{\text {ref }}$ and $\mathrm{k}_{\mathrm{ult}}^{0}$ Definition. 


\section{CAlCUlation OF ULTIMATE BEARING CAPACITY}

The results for the ring footing of internal to external radius ratio (ri/ro) of 0.2 placed on (soft-medium-strong) multilayered soil and ultimate bearing capacity is calculated, for $0,1,2,3,4,5,6,7$ reinforcement layers of geogrid. Fig. 5 shows the variation of ultimate bearing capacity with no. of reinforcement layers at $(\mathrm{ri} / \mathrm{ro}=0.2)$.

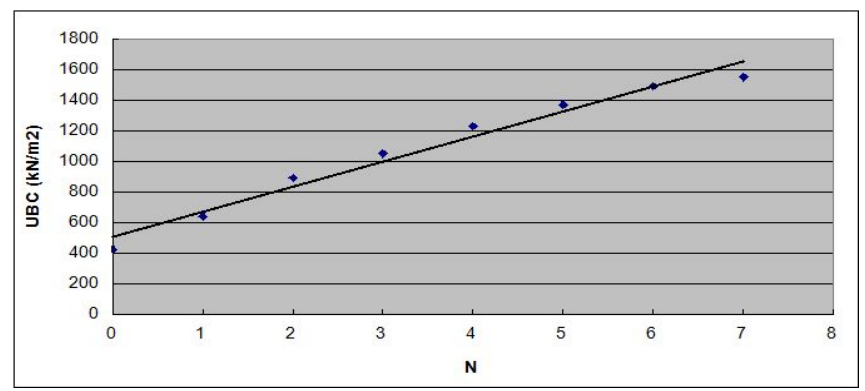

Figure 5: Variation of Ultimate Bearing Capacity with No. of Reinforcement Layers at (ri/ro $=0.2)$

The results for the ring footing of internal to external radius ratio (ri/ro) of 0.2 placed on (medium-strong-soft) multilayered soil and ultimate bearing capacity is calculated, for $0,1,2,3,4,5,6,7$ reinforcement layers of geogrid. Fig.6 shows the variation of ultimate bearing capacity with no. of reinforcement layers at $(\mathrm{ri} / \mathrm{ro}=0.2)$.

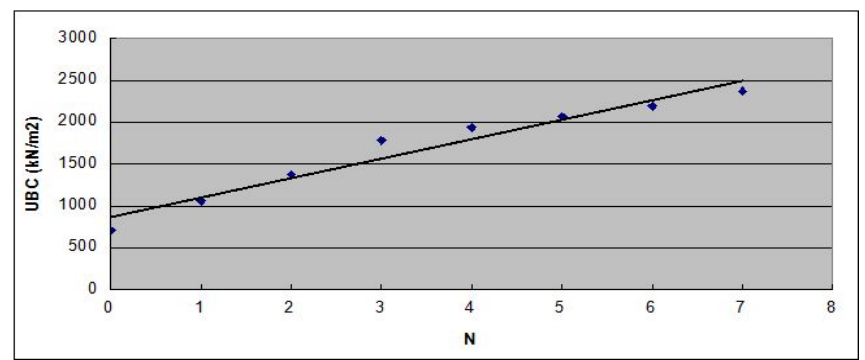

Figure 6: Variation of Ultimate Bearing Capacity with No. of Reinforcement Layers at $(\mathrm{ri} / \mathrm{ro}=0.2)$

The results for the ring footing of internal to external radius ratio (ri/ro) of 0.4 placed on (soft-medium-strong) multilayered soil and ultimate bearing capacity is calculated, for $0,1,2,3,4,5,6,7$ reinforcement layers of geogrid. Fig.7 shows the variation of ultimate bearing capacity with no. of reinforcement layers at (ri/ro $=0.4)$.

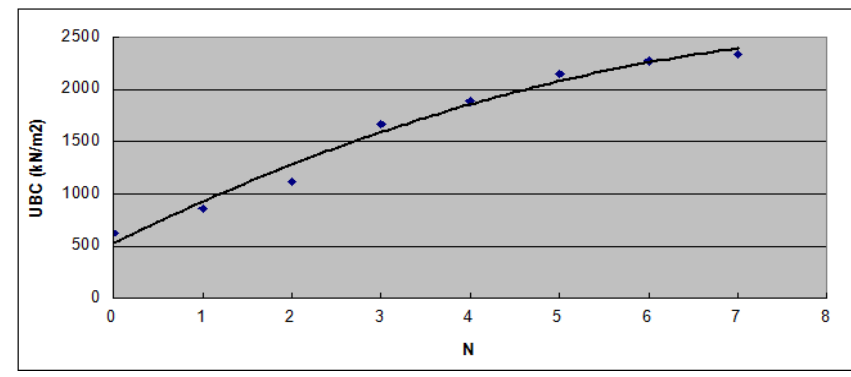

Figure 7: Variation of ultimate bearing capacity with no. Of reinforcement layers at $(\mathrm{ri} / \mathrm{ro}=0.4)$
The results for the ring footing of internal to external radius ratio (ri/ro) of 0.4 placed on (medium-strong-soft) multilayered soil and ultimate bearing capacity is calculated, for $0,1,2,3,4,5,6,7$ reinforcement layers of geogrid. Fig. 8 shows the variation of ultimate bearing capacity with no. of reinforcement layers at (ri/ro $=0.4$ ).

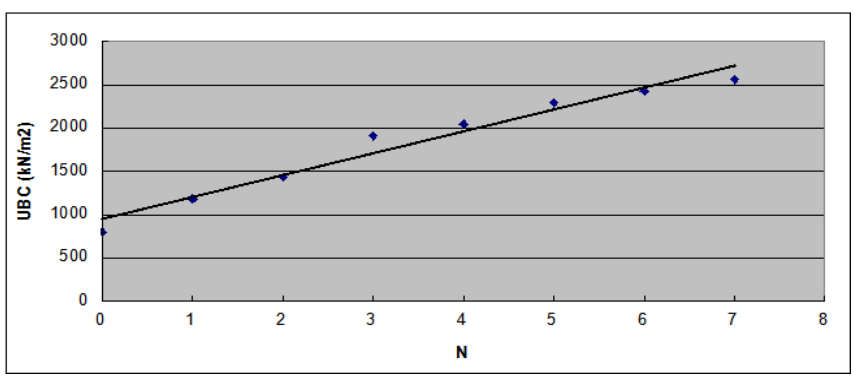

Figure 8: Variation of ultimate bearing capacity with no. of reinforcement layers at (ri/ro $=0.4)$.

The results for the ring footing of internal to external radius ratio (ri/ro) of 0.6 placed on (soft-medium-strong) multilayered soil and ultimate bearing capacity is calculated, for $0,1,2,3,4,5,6,7$ reinforcement layers of geogrid. Fig. 9 shows the variation of ultimate bearing capacity with no. of reinforcement layers at $(\mathrm{ri} / \mathrm{ro}=0.6)$.

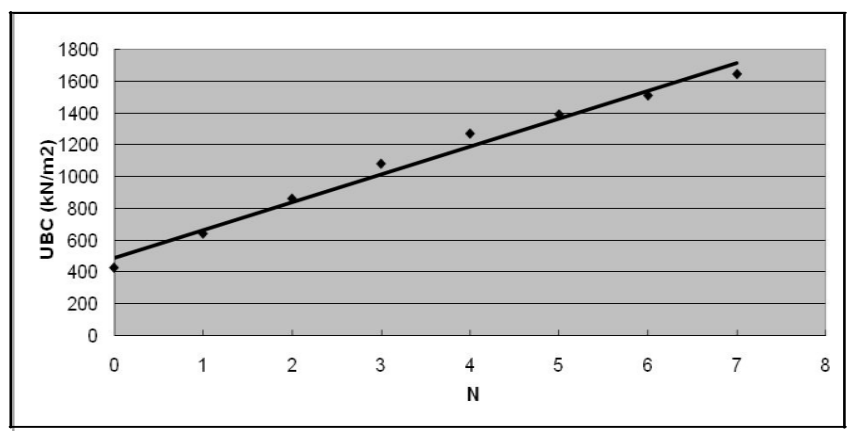

Figure 9: Variation of ultimate bearing capacity with no. Of reinforcement layers at (ri/ro $=0.6)$.

The results for the ring footing of internal to external radius ratio (ri/ro) of 0.6 placed on (medium-strong-soft) multilayered soil and ultimate bearing capacity is calculated, for $0,1,2,3,4,5,6,7$ reinforcement layers of geogrid. Fig. 10 shows the variation of ultimate bearing capacity with no. of reinforcement layers at $(\mathrm{ri} / \mathrm{ro}=0.6)$.

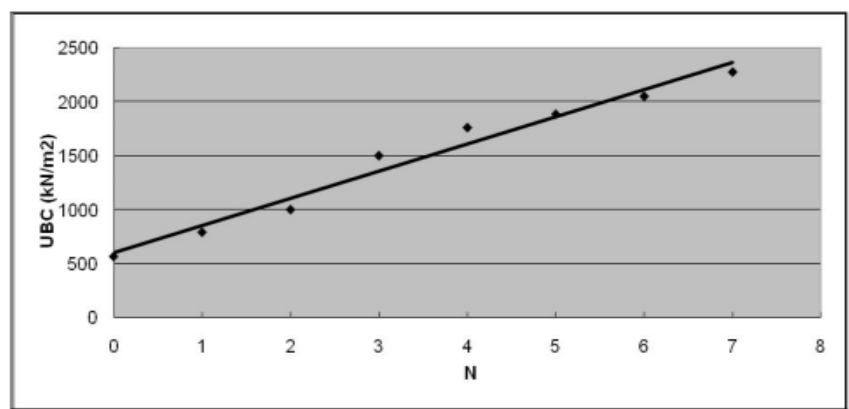

Figure 10: Variation of ultimate bearing capacity with no. Of reinforcement layers at $(\mathrm{ri} / \mathrm{ro}=0.6)$ 
The results for the ring footing of internal to external radius ratio (ri/ro) of 0.8 placed on (soft-medium-strong) multilayered soil and ultimate bearing capacity is calculated, for $0,1,2,3,4,5,6,7$ reinforcement layers of geogrid. Fig. 11 shows the variation of ultimate bearing capacity with no. of reinforcement layers at $($ ri $/$ ro $=0.8)$.

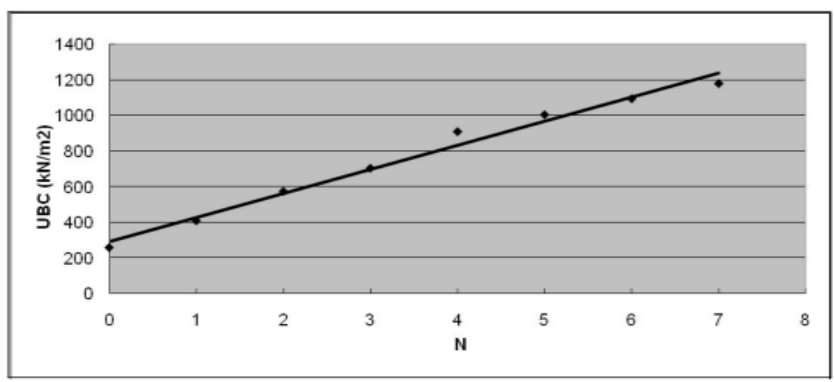

Figure 11: Variation of ultimate bearing capacity with no. of reinforcement layers at $(\mathrm{ri} / \mathrm{ro}=0.8)$.

The results for the ring footing of internal to external radius ratio (ri/ro) of 0.8 placed on (medium-strong-soft) multilayered soil and ultimate bearing capacity is calculated, for $0,1,2,3,4,5,6,7$ reinforcement layers of geogrid. Fig. 12 shows the variation of ultimate bearing capacity with no. of reinforcement layers at (ri/ro $=0.8)$.

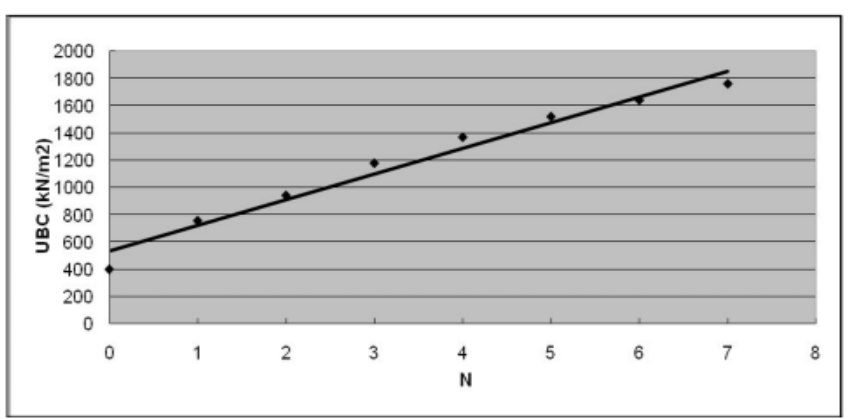

Figure 12: Variation of ultimate bearing capacity with no. of reinforcement layers at (ri/ro $=0.8)$.

\section{CALCULATION FOR SETTELMENT REDUCTION RATIO}

The results for the ring footing of internal to external radius ratio (ri/ro) of 0.2 placed on (soft-mediumstrong) multilayered soil and settlement reduction ratio is calculated, for $0,1,2,3,4,5,6,7$ reinforcement layers of geogrid. Fig. 13 shows the variation of settlement reduction ratio with no. of reinforcement layers at (ri/ro $=0.2)$.

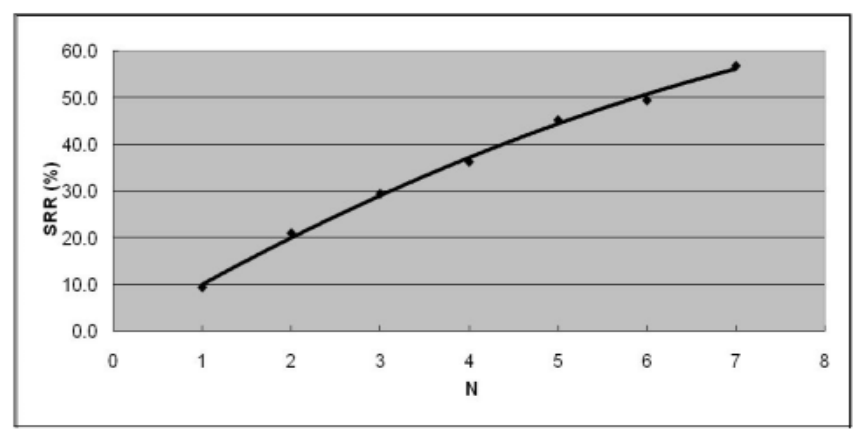

Fig 13: Variation of settlement reduction ratio with no. of reinforcement layers at $(\mathrm{ri} / \mathrm{ro}=0.2)$.
The results for the ring footing of internal to external radius ratio (ri/ro) of 0.2 placed on (medium-strong-soft) multilayered soil and settlement reduction ratio is calculated, for $0,1,2,3,4,5,6,7$ reinforcement layers of geogrid. Fig. 14 shows the variation of settlement reduction ratio with no. of reinforcement layers at $(\mathrm{ri} / \mathrm{ro}=0.2)$.

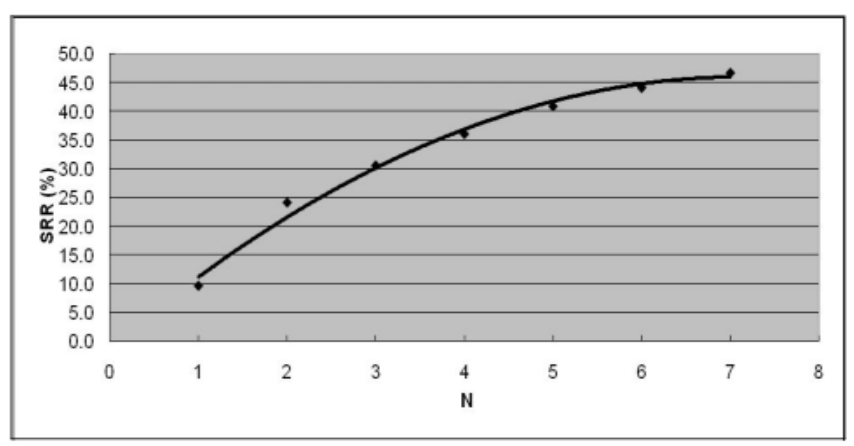

Fig 14: Variation of settlement reduction ratio with no. of reinforcement layers at $(\mathrm{ri} / \mathrm{ro}=0.2)$.

The results for the ring footing of internal to external radius ratio (ri/ro) of 0.4 placed on (soft-medium-strong) multilayered soil and settlement reduction ratio is calculated, for $0,1,2,3,4,5,6,7$ reinforcement layers of geogrid. Fig. 15 shows the variation of settlement reduction ratio with no. of reinforcement layers at $(\mathrm{ri} / \mathrm{ro}=0.4)$.

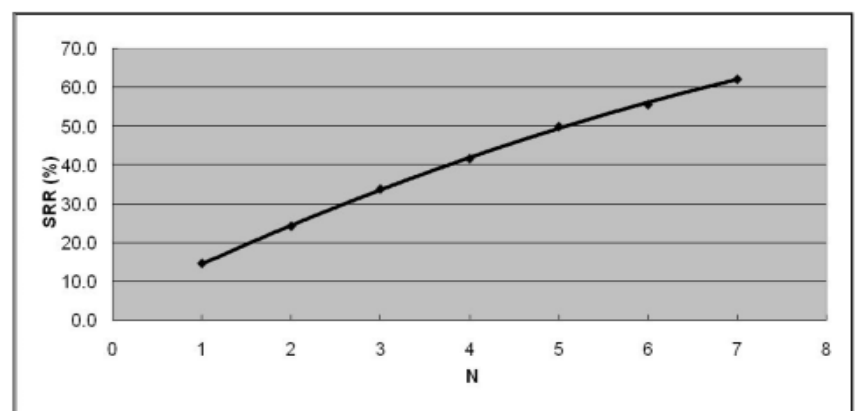

Fig 15: Variation of settlement reduction ratio with no. of reinforcement layers at $(\mathrm{ri} / \mathrm{ro}=0.4)$.

The results for the ring footing of internal to external radius ratio (ri/ro) of 0.4 placed on (mediumstrong-soft) multilayered soil and settlement reduction ratio is calculated, for $0,1,2,3,4,5,6,7$ reinforcement layers of geogrid. Fig. 16 shows the variation of settlement reduction ratio with no. of reinforcement layers at (ri/ro $=$ $0.4)$.

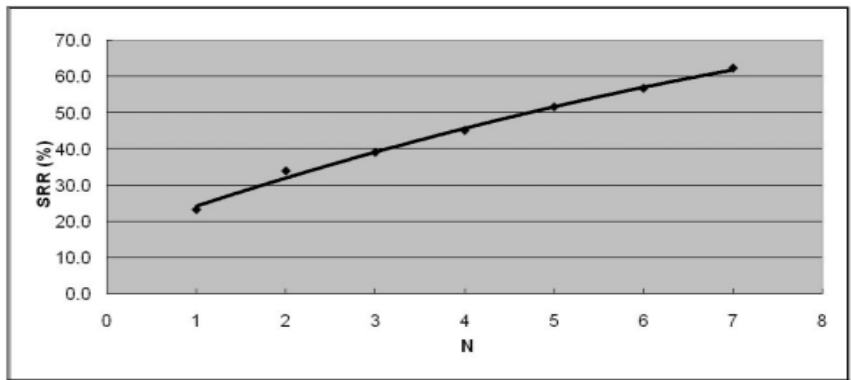

Fig 16: Variation of settlement reduction ratio with no. of reinforcement layers at $(\mathrm{ri} / \mathrm{ro}=0.4)$. 
The results for the ring footing of internal to external radius ratio (ri/ro) of 0.6 placed on (soft-mediumstrong) multilayered soil and settlement reduction ratio is calculated, for $0,1,2,3,4,5,6,7$ reinforcement layers of geogrid. Fig. 17 shows the variation of settlement reduction ratio with no. of reinforcement layers at (ri/ro $=0.6)$.

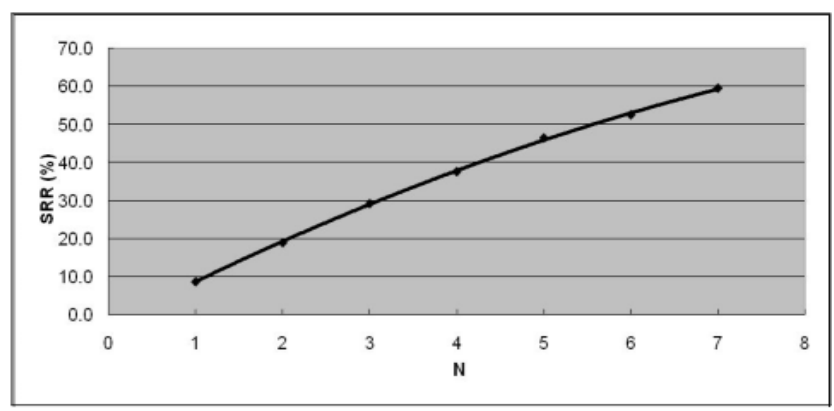

Fig 17: Variation of settlement reduction ratio with no. of reinforcement layers at $(\mathrm{ri} / \mathrm{ro}=0.6)$.

The results for the ring footing of internal to external radius ratio (ri/ro) of 0.6 placed on (mediumstrong-soft) multilayered soil and settlement reduction ratio is calculated, for $0,1,2,3,4,5,6,7$ reinforcement layers of geogrid. Fig. 18 shows the variation of settlement reduction ratio with no. of reinforcement layers at (ri/ro = $0.6)$.

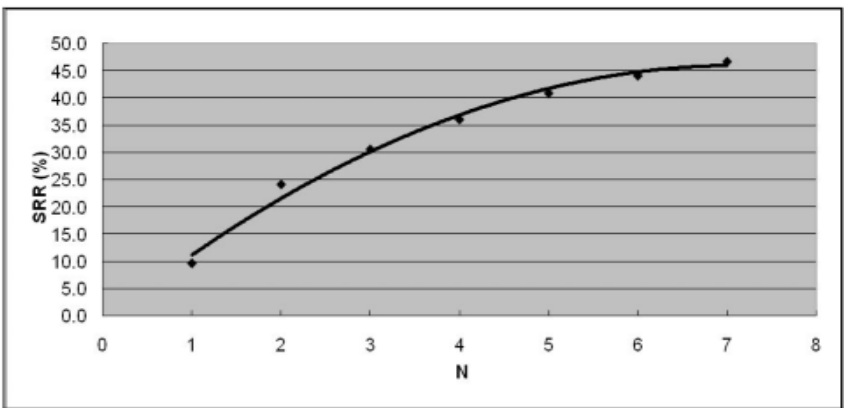

Fig 18: Variation of settlement reduction ratio with no. of reinforcement layers at $(\mathrm{ri} / \mathrm{ro}=0.6)$.

The results for the ring footing of internal to external radius ratio (ri/ro) of 0.8 placed on (soft-mediumstrong) multilayered soil and settlement reduction ratio is calculated, for $0,1,2,3,4,5,6,7$ reinforcement layers of geogrid. Fig. 19 shows the variation of settlement reduction ratio with no. of reinforcement layers at (ri/ro $=0.8$ ).

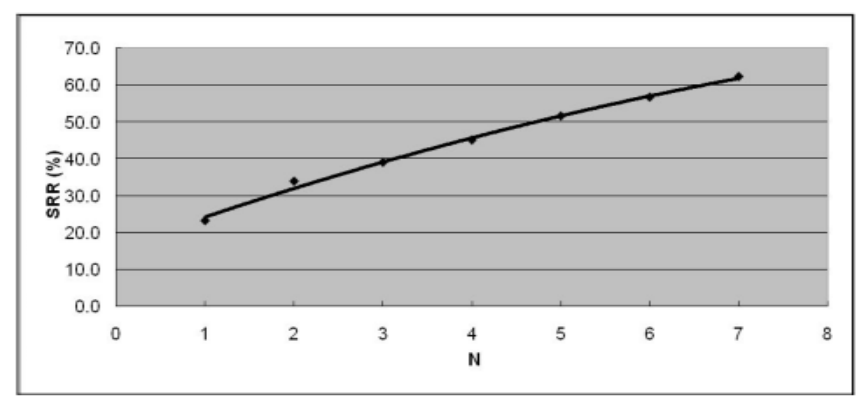

Fig 19: Variation of settlement reduction ratio with no. of reinforcement layers at $(\mathrm{ri} / \mathrm{ro}=0.8)$.
The results for the ring footing of internal to external radius ratio (ri/ro) of 0.8 placed on (mediumstrong-soft) multilayered soil and settlement reduction ratio is calculated, for $0,1,2,3,4,5,6,7$ reinforcement layers of geogrid. Fig. 20 shows the variation of settlement reduction ratio with no. of reinforcement layers at (ri/ro $=$ $0.8)$.

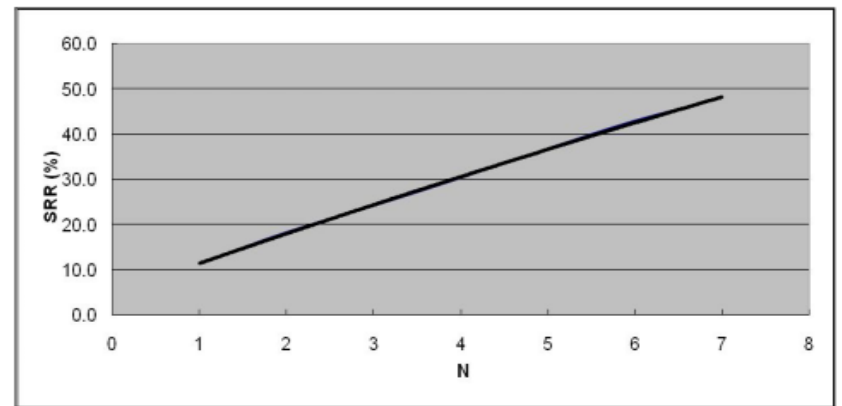

Fig 20: Variation of settlement reduction ratio with no. of reinforcement layers at $(\mathrm{ri} / \mathrm{ro}=0.8)$.

\section{CONCLUSION}

- It is found that the bearing capacity increases as the inner radius to outer radius ratio increases upto 0.4 and afterwards the bearing capacity decreases.

- The bearing capacity of ring footing is reduced if a weaker layer is present below a stronger crust. The limit load is affected by the depth of the layers.

\section{REFERENCES}

[1] Sawwaf, M. El., and Nazir, A. (2012). "Behavior of eccentrically loaded small scale ring Footings resting on reinforced layered soil." Journal of Geotechnical and Geoenvironmental Engineering., ASCE.138(3).

[2] Benmebarek, S., Remadna, M. S.,Benmebarek, N., Belounar, N. (2012)."Numerical evaluation of the bearingcapacity factor $\mathrm{N} \gamma$ of ring footing." Journal of Computers and Geotechnics.,ELSEVIER., 44, 132138.

[3] Moayed, R. Z., Rashidian, V., and Izadi, E.(2012). "Evaluation of bearing capacity of Ring footing on two layered soil." World Academy of science Engineeering and technology.

[4] Boushehrian, A.H., and Hataf, N. (2010). "Bearing capacity of ring footing on reinforced clay." 12 th International conference of International Association for Computer Methods and Advances in Geomechanics., IACMAG. 1-6.

[5] Choobbasti, A. J., Hesami, S., Pirzadeh, S., Farrokhzad, F., and Zahmatkesh, A. (2010). "Numerical evaluation of bearing capacity and settlement of ring footing; case study of kazeroon cooling tower." IJRAS, August 2010.

[6] Mehrjardi, Gh. T. (2008). "Bearing Capacity and settlement of ring footings." The 14th World Conference on Earthquake Engineering., October 1217, 2008, Beijing, China. 
[7] Zhao, L., Wang, J. H. (2008). "Vertical bearing capacity for ring footings." Journal of Computers an Geotechnics., ELSEVIER, 35, 292-304.

[8] Zhu, F., Eng, B., and Eng, M. (1998). "Centrifuge modelling and numerical analysis of bearing capacity of ring foundations on sand." National Library of Canada.

[9] Ismael, N. F. (1996) "Loading test on circular and ring plates in very dense cemented sands." Journal of Geotechnical Engineering., ASCE, 122(4), April.

[10] Beckerand, D., Lo, K. Y. (1979). " Settlement and load transfer of ring foundation for tower silos." Canadian agricultural engineering., 21(2), December.

[11] Snodi, L. N. (2010). "Ultimate bearing capacity of ring footing on sand." Wasit Journal for science and Medicine., 1, (7180).

[12] Turnbull, J. E., Jackson, H. A., and Lowe, D. (1979). "Reinforced extended ring foundations for topunloading concrete tower silos." Canadian Agriculture Engineering,.22(2), December.

[13] Ghosh., Priyanka., Sharma., and Anirudh. (2010). "Interference effect of two nearby square and rectangular footings." Indian Geotechnical Conference GEOtrendz., December 16-18.

[14] Maheshwari, K. V., Desai, A. K., and Solanki, C. H. (2010). "Model footing tests on fiber reinforced soil." Proceedings of Indian Geotechnical Conference., GEOtrendz, December 16-18.

[15] Nagaraj, T. K., Ullagaddi, P. B. (2010). "Experimental study on load settlement behavior of sand foundations." Proceedings of Indian Geotechnical Conference., GEOtrendz, December 16-18.

[16] Wajeeh Mohanmed, T. P., and Ilamparuthi, K. (2010). "Performance of footing on Sand Bed with and without reinforcement." Proceedings of Indian Geotechnical Conference., GEOtrendz, December 16-18.

\section{BIOGRAPHIES}

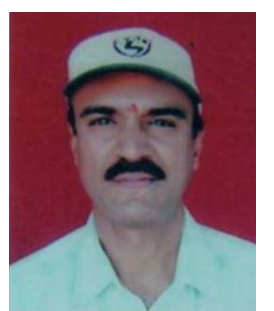

Dr.A.I.Dhatrak, Professor, GCOE Amravati.Experience 27 years teaching to UG and $\mathrm{P}$ G. Research paper Published in International and National Journals more than 30 papers. Visited Foreign Countries 1.Singapore 2. China 3. Thailand \& Guided M.Tech Students more than 10 .

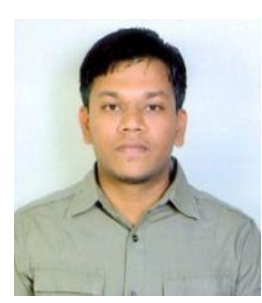

Mr. Pankaj C. Mishra, M.Tech

Geotechnical Engineering, Student, Government College of Engineering Amravati. 